\title{
EDUCAÇÃO FÍSICA E SAÚDE MENTAL: UMA ANÁLISE DA ROTINA DE TRABALHO DOS PROFISSIONAIS DOS CAPS DE GOIÂNIA
}

\author{
EDUCACIÓN FÍSICA Y SALUD MENTAL: UN ANÁLISIS DE LA RUTINA DE \\ TRABAJO DE LOS PROFESIONALES DE LOS CAPS DE GOIÂNIA
}

PHYSICAL EDUCATION AND MENTAL HEALTH: AN ANALYSIS OF THE DAILY ROUTINE OF PE TEACHERS WORKING AT GOIÂNIA'S CAPS

Roberto Pereira Furtado*, Ranulfo Cavalari Neto*, Gleyson Batista Rios ${ }^{\star *}$, Jéssica Félix Nicácio Martinez* Marcos Flávio Mércio de Oliveira*

Palavras-chave

Serviços de Saúde Mental.

Educação Física

Trabalho.
Resumo: $O$ objetivo da pesquisa foi compreender a rotina de trabalho dos professores de Educação Física nos Centros de Atenção Psicossocial (CAPS), com a identificação de suas ações cotidianas e das práticas que dão identidade à Educação Física. A metodologia se desenvolveu a partir de pesquisa de campo de caráter exploratório, realizada em sete CAPS da cidade de Goiânia/GO, com observação da rotina semanal de trabalho de 17 professores de Educação Física. Como resultado, identificamos duas categorias interligadas que auxiliam na compreensão da rotina do trabalho: o cuidado terapêutico, com $63,8 \%$, e o planejamento, organização e avaliação do cuidado terapêutico, com $36,2 \%$. A maior parte das oficinas terapêuticas envolvia práticas corporais, o que demonstra que a identidade da Educação Física encontra-se contemplada.
Palabras clave Servicios de Salud Mental. Educación Física. Trabajo
Resumen: El objetivo de esta investigación ha sido comprender la rutina de trabajo de los profesores de Educación Física en los Centros de Atención Psicosocial (CAPS), a través de la identificación de sus acciones cotidianas y de las prácticas que dan identidad a la Educación Física. La metodología se ha basado en un estudio exploratorio en 7 CAPS de la ciudad de Goiânia, Goiás, con observación de la rutina semanal de trabajo de 17 profesores de Educación Física. Como resultado, identificamos dos categorías interconectadas que auxilian en la comprensión de la rutina de trabajo: el cuidado terapéutico, con un $63,8 \%$ y la planificación, organización y evaluación del cuidado terapéutico, con un $36,2 \%$. La mayoría de los talleres terapéuticos involucraban prácticas corporales, lo que demuestra que la identidad de la Educación Física está contemplada.
Abstract: This study aims to understand the work routine of Physical Education teachers at Psycho-Social Care Centers (CAPS), identifying their every day actions and the practices that establish the identity of Physical Education. The methodology was based on an exploratory study held at 7 CAPS in Goiânia, state of Goiás, observing the weekly routine of 17 Physical Education teachers. As a result, we found two interconnected categories that help to understand the work routine: therapeutic care, with $63.8 \%$, and planning, organization and evaluation of therapeutic care, with $36.2 \%$. Most therapeutic workshops involved bodily practices, demonstrating that Physical Education's identity is covered.

* Universidade Federal de Goiás. Goiânia, GO, Brasil.

E-mail: cremerroberto@hotmail.com

** Secretaria Municipal de Saúde de Goiânia. Goiânia, GO, Brasil. E-mail: gleysonrios@gmail.com

Recebido em: 12-02-2016 Aprovado em: 25-06-2016 (c) (1) (8) Licence 


\section{INTRODUÇÃO}

A crescente demanda por profissionais de Educação Física em serviços especializados de saúde exige que a formação e a atuação profissional observem os princípios e diretrizes do Sistema Único de Saúde (SUS). Entre as diversas possibilidades de intervenção profissional, a saúde mental tem se apresentado como um potencial campo de trabalho para a Educação Física. No Brasil, a proposta de atenção e cuidado na saúde mental está estruturada a partir da Rede de Atenção Psicossocial (RAPS), com os Centros de Atenção Psicossocial (CAPS) como serviço estratégico de articulação. Os CAPS acolhem pessoas com transtornos mentais e/ou com necessidades decorrentes do uso de álcool e outras drogas. São responsáveis por organizar a RAPS e regular sua porta de entrada (BRASIL, 2004). De acordo com Costa-Rosa (2000), essa nova estratégia tem sido construída a partir do processo de Reforma Psiquiátrica, com o intuito de substituição do modelo manicomial, centrado nos hospitais psiquiátricos.

Algumas produções teóricas sobre a Educação Física nos CAPS começaram a ser publicadas mais recentemente, principalmente como resultado de relatos de experiências ou estudos de caso ocorridos em várias regiões do país. Como exemplos, relacionamos abaixo algumas das pesquisas identificadas em nossa revisão de literatura.

Guimarães et al. (2012) apresentam um relato dos CAPS de São João Del-Rei/MG, onde foram desenvolvidas atividades de lutas, danças e esportes adaptados com pessoas com transtornos mentais. Nos CAPS de Recife/PE, foram investigados 12 profissionais de Educação Física em relação à análise de seu processo de trabalho (LEONIDIO et al., 2014). Nos CAPS de Porto Alegre/RS, destacamos a pesquisa de Wachs (2008), que se concentrou nos sentidos presentes no CAPS a respeito da presença de profissionais de Educação Física e suas práticas (WACHS, 2008). Buscando analisar a inserção da Educação Física na equipe multiprofissional, foi realizada uma pesquisa no CAPS de Coari/AM (MIRANDA; FREIRE; OLIVEIRA, 2011). Sobre a Rede de Atenção Psicossocial de Aracaju/SE, identificamos uma pesquisa que visou problematizar o corpo no cuidado em saúde mental (MACHADO, 2011) e outra realizada no CAPS de Aracaju/SE que abordou o papel da Educação Física no cuidado em saúde mental (LIRIO, 2011). No Espírito Santo, Machado (2015) desenvolveu uma pesquisa com o intuito de analisar o trabalho desenvolvido pelos professores de Educação Física em dois CAPS. Por fim, citamos pesquisa realizada nos CAPS de Goiânia com o objetivo de analisar as principais características da intervenção profissional da Educação Física (FURTADO et al., 2015).

Com o intuito de contribuir com reflexões sobre essa nova realidade, realizamos uma pesquisa de campo nos CAPS em Goiânia/GO, buscando compreender o papel desempenhado pelo professor de Educação Física. O objetivo foi compreender como se caracterizavam suas rotinas de trabalho e como as práticas corporais estão presentes nessa rotina. Tal compreensão se faz relevante pois esse campo de trabalho tem sido ocupado pela Educação Física, mas ainda é pouco tematizado nos cursos de formação e muitos profissionais de Educação Física se sentem inseguros em sua inserção inicial, como explicam Furtado et al. (2015).

\section{METODOLOGIA}

A investigação constituiu-se como uma pesquisa de campo de caráter exploratório. ${ }^{1}$ A pesquisa foi realizada na cidade de Goiânia, com os profissionais de Educação Física que trabalhavam nos CAPS do município. A quantidade de CAPS e de profissionais de Educação 
Física foi identificada através de levantamento de dados junto à Secretária Municipal de Saúde. Durante a pesquisa, realizada no ano de 2014, havia dez CAPS na cidade de Goiânia (quatro CAPSad, cinco CAPS II, um CAPSi) ${ }^{2}$ com 21 profissionais de Educação Física concursados em atividade.

A pesquisa foi aprovada pelo Comitê de Ética da Universidade Federal de Goiás e autorizada pela Secretaria Municipal de Saúde de Goiânia/GO. Teve como critério de inclusão: a) atuar como profissional de Educação Física pela Secretaria Municipal de Saúde nos CAPS; e b) assinar o Termo de Consentimento Livre e Esclarecido (TCLE). 0 contato com os profissionais foi realizado no próprio local de trabalho, onde foram convidados a participar da pesquisa e a assinarem o TCLE.

A coleta de dados foi realizada no mês de maio de 2014 após confirmação de normalidade do serviço durante a semana em que seria realizada a pesquisa e a certeza da presença do profissional no local de trabalho. Cada profissional foi acompanhado diariamente durante toda sua jornada de trabalho no período contínuo de uma semana. Dessa forma, cada profissional que aceitou participar da pesquisa foi acompanhado de segunda a sextafeira. Considerando esses elementos, a pesquisa foi desenvolvida em sete CAPS e com 17 professores de Educação Física, o que representa $81 \%$ do total.

Como técnica e instrumento de coleta de dados, utilizamos um roteiro estruturado como diário de campo, o qual foi preenchido durante as observações da rotina de trabalho dos professores investigados. Para cada atividade realizada e profissional observado, foi utilizado um roteiro diferente, ainda que mais de um profissional estivesse trabalhando simultaneamente em um mesmo CAPS. Ao final, obtivemos 105 roteiros preenchidos.

A análise de dados foi desenvolvida a partir da técnica de análise de conteúdo em conformidade com as etapas sugeridas por Minayo (2009). Após reunirmos a totalidade dos roteiros preenchidos, realizamos leitura do conjunto dos dados coletados, buscando identificar seus núcleos centrais de sentido. Nesse processo de delimitação dos dados empíricos chegamos a duas categorias principais: i) cuidado terapêutico e ii) planejamento, organização e avaliação do cuidado terapêutico. A análise foi confrontada e articulada com os referenciais teóricos e quando necessário foi apresentada com o auxílio da estatística descritiva.

\section{RESULTADOS E DISCUSSÃO}

\subsection{A rotina de trabalho do profissional de Educação Física}

De acordo com as orientações do Ministério da Saúde, os CAPS devem se apoiar em uma perspectiva de atenção psicossocial que extrapola a clínica tradicional, caracterizada principalmente por ações restritas a consultas médicas, psicológicas e uso de medicamentos. Devem, portanto, compreender outras dimensões igualmente importantes para a saúde e produção da vida, consolidando uma clínica ampliada. As ações podem ser individuais, em

2 Os CAPS se diferenciam entre si pelo tamanho da população de referência e pela especificidade da demanda atendida. Na cidade na qual esta pesquisa foi realizada havia CAPSad, CAPS II e CAPSi. Conforme a Portaria GM 3088/2011, o CAPS II atende pessoas com transtornos mentais graves e persistentes, podendo também atender pessoas com necessidades decorrentes do uso de crack, álcool e outras drogas, conforme a organização da rede de saúde local, indicado para municípios com população acima de setenta mil habitantes. Já o CAPSad atende adultos ou crianças e adolescentes, considerando as normativas do Estatuto da Criança e do Adolescente, com necessidades decorrentes do uso de crack, álcool e outras drogas. Por fim, o CAPSi atende crianças e adolescentes com transtornos mentais graves e persistentes e os que fazem uso de crack, álcool e outras drogas, indicado para municípios ou regiões com população acima de 150 mil habitantes. Todas essas modalidades possuem funcionamento de segunda-feira a sexta-feira, das 8 às 18 horas. 
grupos, destinadas às famílias ou comunitárias e desenvolvidas em forma de psicoterapias, tratamento medicamentoso, atendimento a grupo de familiares, visitas domiciliares, atividades comunitárias (como feiras, festa junina, campeonatos esportivos, passeios), atividades de suporte social (como atividades de lazer, encaminhamentos para rede de ensino, obtenção de documentos), além de oficinas culturais, oficinas terapêuticas específicas, entre outros (BRASIL, 2004).

Cadore e Beck (2011) defendem que o processo terapêutico nos CAPS pode ser compreendido a partir de duas grandes dimensões: a política e a clínica. Em relação à dimensão política, os autores exemplificam com as ações de cunho social, como reinserção social, cidadania e a busca pela construção da autonomia do usuário. Já na dimensão clínica encontram-se as condutas terapêuticas que levam em consideração a singularidade do usuário visando promover a saúde mental. Essas duas dimensões são chamadas por Ramminger e Brito (2011) de cuidado clínico e reabilitação psicossocial.

Para fugir da dicotomia que prioriza uma ou outra dimensão, a noção de clínica ampliada abarca ambas, visando "[...] superar a clínica tradicional e a dissociação entre reabilitação psicossocial de um lado e clínica de outro. Para isso propõe uma redefinição do objeto, do objetivo e dos meios de trabalho de assistência individual, familiar e de grupos" (FERREIRA NETO, 2008, p. 112). Tais compreensões estão de acordo com a proposta de oferecer tratamento terapêutico assumindo um papel estratégico na organização da RAPS, "[...] realizando acompanhamento clínico e a reinserção social dos usuários pelo acesso ao trabalho, lazer, exercício dos direitos civis e fortalecimento dos laços familiares e comunitários" (BRASIL, 2004, p. 13, grifo nosso).

A conceituação das dimensões clínicas e políticas que envolvem a clínica ampliada é fundamental para a compreensão do trabalho nos CAPS. Entretanto, avaliamos que para analisar a rotina do trabalho seria necessário acrescentar outras duas dimensões do processo de trabalho, identificadas a partir das observações realizadas. Trata-se de uma diferenciação didática entre duas categorias de atividades que estão relacionadas entre si, sendo a primeira denominada de cuidado terapêutico e a segunda de Planejamento, organização e avaliação do cuidado terapêutico.

Gelsimar José Machado (2015), a realizar estudo acompanhando a rotina de profissionais de Educação Física em dois CAPS, apresenta uma descrição das atividades que se assemelha a nossa construção categorial. De acordo com o autor, o professor de Educação Física atua nas seguintes funções: "[...] atenção diária, caminhada, yoga, ginástica, horta, profissional de referência e comissão da atenção diária" (MACHADO, 2015, p. 60). Tais funções situam-se dentro da categoria que estamos denominando de cuidado terapêutico. Acrescenta ainda que "o professor também participa de outras questões: Reunião de Equipe, Visita Domiciliar, Reunião de Território, Reunião de Saúde Mental, Reunião sobre Formação Profissional" (MACHADO, 2015 , p. 60). Esse outro conjunto de atividades faz parte do que estamos denominando como processo de planejamento, organização e avaliação do cuidado terapêutico.

Toda a organização do cuidado terapêutico se inicia a partir do acolhimento ao usuário. Geralmente, no momento do primeiro acolhimento, um profissional ou uma pequena equipe passa a ser referência para o usuário acolhido. A partir daí, há uma série de outras atividades relacionadas com o planejamento, organização e avaliação do cuidado terapêutico. Essas atividades são sistematizadas em um projeto terapêutico destinado a cada novo usuário 
acolhido pelo CAPS. Nesse projeto terapêutico singular (PTS), as necessidades particulares de cada usuário são consideradas e alguns grupos terapêuticos ou outras formas de intervenção são indicados para que ele participe.

Sobre o projeto terapêutico, Leal e Munõz (2014), argumentando na perspectiva da clínica ampliada, nos lembram que várias ações são importantes além da indicação de medicamentos. A primeira delas é o estabelecimento de uma relação de confiança da equipe de profissionais com o usuário e sua família. Nessa busca pela construção de um vínculo de confiança, promover encontros é algo fundamental. Tais encontros são estabelecidos inicialmente com a família e o usuário nas atividades de acolhimento, mas também devem ser promovidos em forma de visitas domiciliares, acompanhamento no território, confraternizações, enfim, momentos em que os vínculos entre a equipe de profissionais e os usuários com suas famílias possam ser fortalecidos. Para tanto, "[...] a forma de abordagem deve ser cuidadosa e respeitosa, oferecendo uma escuta acolhedora e desprovida de expectativas ou prejulgamentos. É importante falar de modo claro com o usuário, explicando-lhe as ações promovidas e as atividades disponíveis" (LEAL; MUNÕZ, 2014, p. 77). Com esse intuito, de estreitar a confiança e o vínculo da equipe com o usuário e sua família, a definição de profissionais que serão terapeutas de referência se faz importante.

É a partir dessas características que a rotina de trabalho é organizada e desenvolvida no cuidado terapêutico e no planejamento, organização e avaliação do cuidado terapêutico. A seguir procuramos detalhar como ambos compõem a rotina de trabalho.

\subsection{A rotina no cuidado terapêutico}

As atividades que envolvem o cuidado terapêutico são aquelas em que há contato direto com o usuário, podendo ser sistematizadas a partir de planejamentos ou serem assistemáticas e eventuais, realizadas por um profissional ou por equipes multiprofissionais.

Em nossas observações, foram identificadas na rotina de trabalho dos professores de Educação Física dos CAPS de Goiânia as seguintes atividades que envolvem o cuidado terapêutico: a) Oficinas Terapêuticas; b) Referente dia; c) Acolhimento; d) Consulta conjunta; e) Passeios; f) Atendimento familiar; g) Visita domiciliar; h) Confraternização. Apresentamos abaixo uma pequena descrição do que é realizado em cada uma das atividades de cuidado terapêutico encontradas na rotina de trabalho do profissional de Educação Física.

a) Oficinas terapêuticas - são estabelecidas por cada CAPS de acordo com o perfil de seus profissionais e com o interesse e necessidade dos usuários, tendo como objetivos a maior integração social e familiar, a manifestação de sentimentos e problemas, o desenvolvimento de habilidades corporais, a realização de atividades produtivas e o exercício coletivo da cidadania (BRASIL, 2004). Muitas oficinas são sistematizadas a partir de conteúdos característicos das profissões presentes nos CAPS. Outras são organizadas em formato de rodas de conversa e utilizam estratégias diversas, como músicas, filmes, conversas sobre assuntos do cotidiano, entre outras ações. O profissional de Educação Física pode estar envolvido em cada uma delas.

b) Referente dia - um profissional é destacado para acompanhar/coordenar a rotina do turno na unidade, identificar usuários que ainda não receberam a atenção necessária, dar apoio em situações de crise, encaminhar casos que precisam estabelecer relações com outros serviços etc. Essa forma de organização foi identificada em apenas um dos CAPS analisados. 
c) Acolhimento - trata-se de receber o usuário, em crise ou não, quando ele acessa o serviço. Pode ser individual ou em grupo, realizado por um ou mais profissionais. Objetiva realizar uma primeira escuta e compreender de forma mais abrangente possível a situação que levou o usuário a procurar o serviço.

d) Consulta conjunta - são atendimentos realizados por categorias profissionais distintas em conjunto. No caso observado, houve apenas uma consulta conjunta que envolveu 0 profissional de Educação Física e o médico.

e) Passeios - são ações com cunho educativo e recreativo. Os usuários são levados a alguma instituição, atividade, parque, museu, viagens ou eventos diversos que acontecem na cidade. São organizados e planejados por cada CAPS, de acordo com as suas possibilidades e escolhas da gestão.

f) Atendimento familiar - são atividades de cunho educativo e terapêutico, desenvolvidas com a finalidade de empoderar os familiares no cuidado aos usuários e de estabelecer uma relação de cuidado terapêutico com os próprios familiares. Mielke et al. (2009, p. 162) explicam que 0 cuidado "[...] envolve também a família do usuário, que é entendida como parte fundamental para a evolução satisfatória do usuário no paradigma psicossocial de atenção à saúde mental".

g) Visita domiciliar - são visitas realizadas às residências dos usuários por demandas judiciais, da família, do usuário, da necessidade do próprio serviço, na realização de busca ativa, ou na tentativa de compreender melhor questões relacionadas ao contexto de moradia do usuário.

h) Confraternização - são atividades com características festivas, organizadas por profissionais e usuários, normalmente relacionadas a datas comemorativas.

Na tabela abaixo, está apresentada a relação dessas atividades com a quantidade de participações de profissionais de Educação Física na semana em que a rotina de cada profissional foi observada. É importante reforçar que acompanhamos cada profissional de Educação Física durante toda a sua jornada de trabalho. Entretanto, simultaneamente, profissionais de outras áreas realizavam outras atividades. Isso significa que a quantidade apresentada na tabela diz respeito apenas às atividades desenvolvidas pelos profissionais de Educação Física, individualmente ou em conjunto com outros profissionais. Mas a quantidade de cada uma dessas atividades nos CAPS é maior, pois outros profissionais também as desempenham.

Tabela 1 - Atividades de cuidado terapêutico

\begin{tabular}{ccc}
\hline Atividades & Quantidade & Percentual \\
\hline Oficinas Terapêuticas & 46 & $69 \%$ \\
Acolhimento & 8 & $12 \%$ \\
Referente Dia & 6 & $9 \%$ \\
Passeios & 3 & $4 \%$ \\
Consulta conjunta & 1 & $1,5 \%$ \\
Confraternização & 1 & $1,5 \%$ \\
Atendimento Familiar & 1 & $1,5 \%$ \\
Visita domiciliar & 1 & $1,5 \%$ \\
\hline Total & 67 & $100 \%$ \\
\hline
\end{tabular}


Entre as atividades de cuidado terapêutico, o profissional de Educação Física está mais envolvido com as oficinas terapêuticas, representando uma proporção aproximada de $69 \%$ do total. Elas foram realizadas em espaços físicos localizados no interior dos CAPS e outras em espaços e instituições do território adscrito, indicando potencial no processo de desinstitucionalização e reinserção social do usuário. Em contrapartida, esse profissional está pouco presente na consulta conjunta e no atendimento familiar, por exemplo. Algumas atividades não são tão recorrentes na rotina dos CAPS, como os passeios, as visitas domiciliares, confraternizações e outras. São atividades realizadas mais esporadicamente, enquanto que as oficinas terapêuticas têm uma programação contínua, geralmente semanal ou até mesmo com mais de uma sessão por semana. As consultas, acolhimentos e atendimentos familiares também são atividades bastante recorrentes.

Em nossas observações, também percebemos que poucas foram as oficinas terapêuticas, além das 46 registradas, que não possuíam profissionais de Educação Física envolvidos. Isso indica que com as oficinas terapêuticas o profissional de Educação Física tem construído maior legitimidade de intervenção.

Embora com menor presença, os passeios, a consulta conjunta, confraternizações, atendimento familiar e a visita domiciliar são importantes atividades que envolvem o cuidado terapêutico. Os passeios contribuem bastante na desinstitucionalização e na construção de estratégias de reinserção social, pois podem viabilizar condições de empoderamento para a apropriação pelos usuários das diversas instituições e práticas presentes na cidade e no território. As consultas conjuntas evidenciam a tentativa de maior aproximação da interdisciplinaridade, fundamental para o cuidado na lógica da clínica ampliada, fortalecendo o princípio da integralidade no cuidado. Evidenciam também que o trabalho nos CAPS possui uma dimensão clínica importante que é indissociável da dimensão política. Nesse ponto, é importante ressaltar que o objetivo dos CAPS perpassa tanto o "[...] cuidado clínico quanto à reabilitação psicossocial de pessoas que sofrem com transtornos mentais severos e persistentes, além da organização da rede de saúde mental em seu território" (RAMMINGER; BRITO, 2011, p. 155).

Entretanto, a pequena quantidade de participações dos profissionais de Educação Física no atendimento familiar e consultas conjuntas, que são atividades que ocorrem em quantidade significativa nas rotinas dos CAPS, suscita questionamentos a respeito dos motivos pelos quais a Educação Física está pouco presente nesses espaços e tão presente em oficinas terapêuticas. Nossa análise não poderia deixar de enfatizar a necessidade da Educação Física se fazer presente em ambas as dimensões que compõem a clínica ampliada. Porém, os dados apresentados levantam dúvidas se isto de fato ocorre, pois a ênfase nas oficinas terapêuticas pode indicar que as contribuições da Educação Física e a forma como essa profissão tem conquistado legitimidade nos CAPS são, prioritariamente, através daquilo que é denominado por Cadore e Beck (2011) de dimensão política do cuidado e, por Ramminger e Brito (2011), de reabilitação psicossocial. Contudo, a dimensão do cuidado clínico pode estar devidamente contemplada nas oficinas terapêuticas, caso elas estejam planejadas e conduzidas observando aspectos indispensáveis ao cuidado clínico, como a necessidade singular dos usuários de acordo com as características dos transtornos diagnosticados. Os limites da coleta de dados desta pesquisa não nos permitem afirmar de forma conclusiva, mas a identificação destas duas possibilidades significa um passo adiante na tentativa de melhor compreender o trabalho do professor de Educação Física nos CAPS. 


\subsection{A rotina no planejamento, organização e avaliação do cuidado terapêutico}

Para que o cuidado terapêutico possa ocorrer, uma série de outras atividades são necessárias envolvendo planejamento, organização e avaliação. Essas atividades são realizadas pelos profissionais individualmente ou em grupo e, geralmente, são desenvolvidas sem o contato direto com o usuário do serviço ou com seus familiares. Partindo de tal definição, foram identificadas na rotina de trabalho dos professores de Educação Física dos CAPS de Goiânia as seguintes atividades relacionadas a essa categoria: a) Evolução de prontuário; b) Reunião de equipe/gestão; c) Reunião de equipe referência; d) Atividades administrativas; e) Referência terapêutica/elaboração do Projeto Terapêutico Singular (PTS).

Apresentamos abaixo uma pequena explicação do significado de cada uma das atividades mencionadas.

a) Evolução de prontuário - ações de registro dos atendimentos realizados. Os prontuários são ferramentas de preenchimento e acesso de toda a equipe multiprofissional, o que permite acompanhar em um só documento todas as atividades realizadas pelo usuário.

b) Reunião de equipe e de gestão - nela são planejadas a organização do processo de trabalho do CAPS, a articulação das ações entre os turnos (matutino e vespertino) e atividades que envolvem toda a equipe. Também são discutidos casos complexos em atendimento ou ainda, como em alguns dos CAPS observados, todos os casos recém-acolhidos. Ocorre semanalmente.

c) Reunião de equipe de referência - momento de organização do processo de trabalho com vistas a planejar ações para os usuários referenciados pela equipe e avaliar o processo de tratamento. Como exemplos temos as discussões de casos e o planejamento de visitas ou ações necessárias para a qualificação do acompanhamento. Não são todos os CAPS que se organizam a partir da equipe de referência.

d) Referência Terapêutica e Projeto Terapêutico Singular (PTS) - atividade desenvolvida por um profissional ou uma equipe que será a referência do usuário nas relações estabelecidas com o serviço. É responsável por acompanhar e avaliar o cuidado terapêutico, além de sistematizar informações que permitam a reavaliação e planejamento contínuo do PTS que é discutido em equipe e pactuado com o usuário.

e) Atividades administrativas - em algumas ocasiões, os profissionais podem desenvolver alguma atividade administrativa auxiliando a gestão do CAPS.

Na tabela a seguir, a relação dessas atividades está apresentada, com a quantidade de ocorrências ao longo de uma semana.

Tabela 2 - Atividades de planejamento, organização e avaliação do cuidado terapêutico

\begin{tabular}{ccc}
\hline Atividades & Quantidade & $\%$ \\
\hline Reunião de equipe e de Gestão & 17 & 44,7 \\
Evolução de prontuário & 16 & 42,1 \\
Reunião de equipe de referência & 3 & 7,9 \\
Atividades administrativas & 1 & 2,6 \\
Referência terapêutica/Projeto terapêutico singular & 1 & 2,6 \\
\hline Total & 38 & 100 \\
\hline
\end{tabular}


As reuniões de equipe e de gestão e as evoluções de prontuário são as duas atividades mais exercidas pelos profissionais no âmbito do planejamento, organização e avaliação do cuidado terapêutico. Essas atividades são importantes e compõem parte significativa da rotina do profissional. Sobre as reuniões, Abuhab et al. (2005, p. 373) explicam que elas "fazem parte do processo de trabalho em saúde, porque contribuem para a troca de informações e vivências do dia a dia, onde se colocam as dificuldades e facilidades na execução do trabalho". Vasconcelos (2010) defende que as reuniões são espaços de difusão da reforma psiquiátrica, pois ativam o imaginário de transformação e, assim, ajudam a aglutinar os profissionais em torno de uma mesma perspectiva de cuidado e amortece as dissonâncias entre as disciplinas. Acrescenta ainda que elas "[...] sinalizam a possibilidade de fomentar, com base em procedimento de ajuste mútuo, a troca de informações sobre os usuários" (VASCONCELOS, 2010, p. 14).

A quantidade significativa de profissionais que estavam em reunião com outros visando planejar, organizar e avaliar as atividades demonstra o esforço em conduzir um trabalho multiprofissional construído coletivamente e, portanto, que pode avançar na direção de ações interdisciplinares. Entretanto, a quantidade de 38 atividades relacionadas com o processo de planejamento, organização e controle do cuidado terapêutico, em um universo total de 105 atividades registradas, indica que aproximadamente $36 \%$ do que é realizado pelo profissional em sua rotina de trabalho encontram-se relacionados a atividades que são preparatórias para o cuidado terapêutico. Essa proporção suscita reflexões a respeito da natureza das atividades desempenhadas pelos profissionais do CAPS e sobre se a quantidade de atividades preparatórias ao cuidado terapêutico não estaria em excesso, diminuindo o tempo de trabalho dedicado ao próprio cuidado dos usuários, que é a finalidade principal do serviço.

\subsection{A rotina de trabalho e a identidade da Educação Física}

Quando analisamos as atividades realizadas pelos professores de Educação Física, percebemos que muitas não estão diretamente relacionadas com o objeto que dá identidade a esse campo acadêmico e profissional. Isso ocorre porque os profissionais devem atuar em todas as dimensões dos CAPS e não apenas naquilo que é imediatamente característico das respectivas profissões. Nos CAPS, assim como em outros serviços de saúde, o processo de trabalho é permeado por ações que extrapolam as identidades profissionais.

Foi apenas nas oficinas terapêuticas que identificamos a presença do objeto da Educação Física manifestando-se através de conteúdos como futebol, futsal, lutas, exercícios físicos/ginásticos, práticas corporais integrativas, dança, praticas corporais de aventura, jogos, brincadeiras, avaliação física, recreação e natação. Na rotina semanal de trabalho dos 17 professores, 36 sessões de oficinas terapêuticas relacionadas com práticas corporais foram realizadas. Em geral, as pesquisas que analisaram o desenvolvimento de oficinas com práticas corporais por profissionais de Educação Física nos CAPS as avaliam positivamente, conforme pode ser identificado em Abib et al. (2010), Lírio (2011), Guimarães et al. (2012) e Gelsimar José Machado (2015).

O total das atividades realizadas na rotina de trabalho semanal dos 17 professores foi de 105 . Dessas, $36(34,3 \%)$ referiam-se a aspectos que estão diretamente vinculados com os conteúdos identificadores do campo e 69 (65,7\%) estavam relacionadas com outras necessidades demandadas pelo serviço. Entre as próprias oficinas terapêuticas, 
foram identificadas dez que não apresentavam vínculo direto com práticas corporais como conteúdo. Dessa forma, nas 67 atividades de cuidado terapêutico, totalizam-se 31 atividades não relacionadas com o objeto da Educação Física e 36 atividades relacionadas com esse objeto. Na categoria de atividades de planejamento, organização e avaliação do cuidado terapêutico foram identificadas 38 atividades, e nenhuma delas está diretamente relacionada com a identidade da Educação Física. A tabela abaixo ajuda a visualizar essas quantidades e proporções:

Tabela 3 - Rotina de trabalho e identidade da Educação Física

\begin{tabular}{ccc}
\hline Atividades & Quantidade & $\%$ \\
\hline 1. Cuidado terapêutico & 67 & 63,8 \\
1.1. Atividades envolvendo praticas corporais & 36 & 34,3 \\
1.2. Outras oficinas terapêuticas & 10 & 9,5 \\
1.3. Outras atividades & 21 & 20 \\
2. Planejamento, organização e avaliação do cuidado terapêutico & 38 & 36,2 \\
\hline Total & 105 & 100 \\
\hline
\end{tabular}

Fonte: Dados da pesquisa.

A participação dos profissionais de Educação Física em oficinas terapêuticas propostas por profissionais de outros campos disciplinares é um desafio a ser enfrentado, pois apenas $\operatorname{dez}(21,7 \%)$ das 46 oficinas terapêuticas foram desenvolvidas com temáticas não relacionadas à Educação Física. Essa proporção é semelhante à apresentada por Nascimento e Galvanese (2009), que identificaram "[...] 41 atividades grupais envolvendo usuários realizadas no território de abrangência do CAPS, das quais 31 eram atividades físicas e/ou de socialização e dez eram atividades culturais" (NASCIMENTO; GALVANESE, 2009, p. 13). Por outro lado, percebemos que a identidade do campo acadêmico profissional está presente na rotina de trabalho do professor de Educação Física de forma significativa, pois representa mais da metade das intervenções de cuidado terapêutico e quase $80 \%$ das oficinas terapêuticas desenvolvidas por esses profissionais.

O processo de trabalho em saúde e, nesse caso, em saúde mental, apresenta uma tensão entre a identidade de cada campo de conhecimento e a necessidade de desvincular-se dessa identidade. Essa tensão não pode ser avaliada simplesmente como algo negativo ou positivo, em uma dualidade que não apreende a realidade concreta. Mas deve ser compreendida como uma tensão necessária, pois a saúde é uma produção social e o cuidado deve considerar a integralidade da produção social da vida. As perspectivas unicamente disciplinares diminuem o potencial do cuidado, pois fragmentam e simplificam a multiplicidade de determinações do processo de saúde e doença, não considerando os nexos entre os diversos determinantes das condições de vida e das condições de saúde e, consequentemente, os nexos entre as diversas ciências e práticas sociais que estudam e transformam tais condições. É nessa perspectiva que consideramos importante destacar que a clínica ampliada, a integralidade no cuidado, a reinserção social e o trabalho interdisciplinar, entre outros importantes princípios da atenção psicossocial, exigem que todos os profissionais desenvolvam atividades que extrapolam 0 âmbito de sua formação inicial. 
Gelsimar José Machado (2015) identificou que os professores dos CAPSad analisados por ele pouco questionaram a realização de intervenções que não estivessem diretamente relacionadas à Educação Física. Segundo o autor, os profissionais "[...] entenderam que no CAPSad as demandas são diferentes e que cada trabalhador deve atuar de múltiplas maneiras de modo a contribuir com o serviço e as pessoas em tratamento" (MACHADO, 2015, p. 120). Na mesma perspectiva, Wachs e Fraga $(2009$, p. 8) afirmam que "[...] o professor de educação física não está no serviço para realizar apenas atividades de esportes, ginástica, dança ou outra prática corporal imediatamente associada a sua especificidade".

A tensão entre a necessidade de manter as identidades profissionais e, ao mesmo tempo, abrir mão dessas identidades, pode provocar tensões também entre os profissionais do serviço, como explica Vasconcelos (2010). No meio acadêmico esse debate também é fecundo, há uma procura do que garantiria a especificidade dos respectivos campos. É por não concordar com essa perspectiva de análise que preferimos nos referir a conteúdos que tradicionalmente compõem a identidade das profissões no lugar de conteúdos específicos de uma profissão, pois a noção de especificidade traz consigo a suposição de exclusividade no uso de práticas culturalmente e historicamente produzidas pela humanidade.

\section{CONSIDERAÇÕES FINAIS}

A tarefa de compreender o papel do profissional de Educação Física nos CAPS está apenas se iniciando. Este artigo se limitou a apresentar as ações cotidianas desse profissional no serviço e a presença de práticas que dão identidade ao campo. Identificamos duas categorias que se relacionam entre si e que nos auxiliaram na compreensão e explicação didática da rotina de trabalho desse profissional: o cuidado terapêutico e o planejamento, organização e avaliação do cuidado terapêutico. Enquanto a primeira categoria envolve as ações que se manifestam no encontro com os usuários e familiares, a segunda aglutina as atividades que sustentam a efetivação do cuidado terapêutico. É importante destacar que os dados de nossa pesquisa se referem a uma realidade específica de uma capital brasileira, não podendo, evidentemente, ser generalizados para demais realidades sem a devida repetição dos procedimentos de pesquisa.

Observamos que a maior parte da rotina de trabalho do profissional de Educação Física está concentrada nas atividades de cuidado terapêutico e, nessas, as oficinas terapêuticas foram as principais, indicando maior legitimidade de intervenção. Todavia, a pouca participação desse profissional em atividades de atendimento familiar e consultas conjuntas pode indicar certo distanciamento da Educação Física em relação à dimensão clínica do cuidado e ao trabalho multiprofissional em atividades dessa natureza.

O número considerável de atividades envolvendo práticas corporais revela a necessidade de identificar quais elementos determinam a seleção dessas práticas e como esses elementos estão relacionados com os princípios da política de saúde mental e com as necessidades dos usuários. Pesquisas com tal finalidade poderiam contribuir para a compreensão de como as intervenções dos profissionais de Educação Física articulam as dimensões clínicas e políticas no cuidado terapêutico nos CAPS. 


\section{REFERÊNCIAS}

ABIB, Leonardo Trápaga et al. Práticas corporais em cena na saúde mental: potencialidades de uma oficina de futebol em um centro de atenção psicossocial de Porto Alegre. Pensar a Prática, v. 13, n. 2, p. 1-15, 2010.

ABUHAB, Deborah et al. 0 trabalho em equipe multiprofissional no CAPS III: um desafio. Revista Gaúcha de Enfermagem, v. 26, n. 3, p. 369-380, dez. 2005.

BRASIL. Ministério da Saúde. Portaria GM № 366, de 19 de fevereiro de 2002. Disponível em: <http:// www.maringa.pr.gov.br/cisam/portaria336.pdf>. Acesso em: 12 nov. 2015.

BRASIL. Ministério da Saúde. Saúde mental no SUS: os centros de atenção psicossocial. Brasília: Ministério da Saúde, 2004. Disponível em: <http://www.ccs.saude.gov.br/saude mental/pdf/sm sus. pdf >. Acesso em: 12 nov. 2015.

CADORE, Carlise; BECK, Carmem Lúcia Calomé. O processo terapêutico em um CAPSad: A visão dos Trabalhadores. In: JORNADA DE PESQUISA EM PSICOLOGIA, 4., 2011. p. 122-129. Disponível em: <http://online.unisc.br/acadnet/anais/index.php/jornada psicologia/issue/view/2>. Acesso em: 25 nov. 2015.

COSTA-ROSA, Abílio. O modo psicossocial: um paradigma das práticas substitutivas ao modo asilar. In: AMARANTE, Paulo (Org.). Ensaios: subjetividade, saúde mental, sociedade. Rio de Janeiro: Editora Fiocruz, 2000. p. 141-168.

FERREIRA NETO, João Leite. Práticas transversalizadas da clínica em saúde mental. Psicologia: Reflexão e Crítica, v. 21, n. 1, p. 110-118, 2008.

FURTADO, Roberto Pereira et al. O trabalho do professor de Educação Física no CAPS: aproximações iniciais. Movimento, v. 21, n. 1, p. 41-52, mar. 2015.

GUIMARÃES, Andrea Carmen et al. A inserção social através de práticas de educação física como medidas interventivas para pacientes psicóticos e neuróticos graves do CAPS de São João del-Rei/ MG. Pesquisas e Práticas Psicossociais, v. 7, n. 2, p. 254-259, dez. 2012.

LEAL, Erotildes Maria; MUÑOZ, Nuria Malajovich. Estratégias de intervenção em saúde mental. In: JORGE, Marco Aurélio Soares; CARVALHO, Maria Cecília de Araújo; SILVA, Paulo Roberto Fagundes da (Org.). Políticas e cuidado em saúde mental: contribuições para a prática profissional. Rio de Janeiro: Editora Fiocruz, 2014. p. 75-98.

LEONÍDIO, Ameliane da Conceição Reubens et al. O profissional de Educação Física no Centro de Atenção Psicossocial: percepção dos limites e potencialidades no processo de trabalho. Pesquisas e Práticas Psicossociais, v. 8, n. 2, p. 159-165, dez. 2014.

LIRIO, Acácia Priscilla. Práticas corporais na saúde mental: um relato de experiência do CAPS AD primavera Aracaju/SE. In: CONGRESSO BRASILEIRO DE CIÊNCIAS DO ESPORTE, 17., CONGRESSO INTERNACIONAL DE CIÊNCIAS DO ESPORTE, 4., 2011. Anais... . Porto Alegre: CBCE, 2011. p. 1- 10. Disponível em: <http://congressos.cbce.org.br/index.php/Conbrace2011/2011/ schedConf/presentations>. Acesso em: 25 nov. 2015.

MACHADO, Dagoberto. Os movimentos na educação física: por uma ética dos corpos. 2011. 113 f. Dissertação (Mestrado) - Programa de Pós-graduação em Educação, Universidade Federal do Rio Grande do Sul, Porto Alegre, 2011.

MACHADO, Gelsimar José. A atuação do professor de educação física nos Centros de Atenção Psicossocial Álcool e Drogas. 2015. 130 f. Dissertação (Mestrado) - Programa de Pós Graduação em Educação Física, Universidade Federal do Espírito Santo, Vitória, 2015. 
MIELKE, Fernanda Barreto et al. O cuidado em saúde mental no CAPS no entendimento dos profissionais. Ciência \& Saúde Coletiva, v. 14, n. 1, p. 159-164, fev.2009.

MINAYO, Maria Cecília de Souza. Ciência, técnica e arte: o desafio da pesquisa social. In: MINAYO, Maria Cecília de Souza. (Org.). Pesquisa social: teoria, método e criatividade. Petrópolis: Vozes, 2009. p. 9-15.

MIRANDA, Elisangela Domingos; FREIRE, Luana Almeida; OLIVEIRA, Adriana Rosmaninho Caldeira. Os desafios da Educação Física no Centro de Atenção Psicossocial de Coari (AM). Saúde e Transformação Social, v. 1, n. 2, p. 163-169, 2011.

NASCIMENTO, Andrea de Fátima; GALVANESE, Ana Teresa Costa. Avaliação da estrutura dos centros de atenção psicossocial do município de São Paulo, SP. Revista Saúde Pública, São Paulo, v. 43, p. 8-15, ago. 2009. Supl. 1.

RAMMINGER, Tatiana; BRITO, Jussara Cruz de. "Cada Caps é um Caps": uma coanálise dos recursos, meios e normas presentes nas atividades dos trabalhadores de saúde mental. Psicologia \& Sociedade, v. 23, p. 150-160, 2011.

VASCONCELLOS, Vinícius Carvalho de. Trabalho em equipe na saúde mental: o desafio interdisciplinar em um CAPS. Revista Eletrônica Saúde Mental Álcool e Drogas, v. 6, n. 1, p. 1-22, jan. 2010.

WACHS, Felipe. Educação física e saúde mental: uma prática de cuidado emergente em Centros de Atenção Psicossocial (CAPS). 2008. 133f. Dissertação (Mestrado) - Programa de Pós Graduação em Ciências do Movimento Humano, Universidade Federal do Rio Grande do Sul, Porto Alegre, 2008.

WACHS, Felipe; FRAGA, Alex Branco. Educação Física em centros de atenção psicossocial. Revista Brasileira de Ciências do Esporte, v. 31, n. 1, p. 93-107, set. 2009. 
Apoio:

Conselho Nacional de Desenvolvimento Científico e Tecnológico 